\title{
Global Phase Synchronization for a Class of Dynamical Complex Networks with Time-Varying Coupling Delays
}

\author{
XinBin Li and Haiyan Jing \\ Key Lab of Industrial Computer Control Engineering of Hebei Province, Yanshan University, \\ Qinhuangdao 066004, China \\ Correspondence should be addressed to Haiyan Jing, 1215449068@qq.com
}

Received 25 September 2010; Accepted 22 November 2010

Academic Editor: S. S. Dragomir

Copyright (C 2010 X. Li and H. Jing. This is an open access article distributed under the Creative Commons Attribution License, which permits unrestricted use, distribution, and reproduction in any medium, provided the original work is properly cited.

\begin{abstract}
Global phase synchronization for a class of dynamical complex networks composed of multiinput multioutput pendulum-like systems with time-varying coupling delays is investigated. The problem of the global phase synchronization for the complex networks is equivalent to the problem of the asymptotical stability for the corresponding error dynamical networks. For reducing the conservation, no linearization technique is involved, but by Kronecker product, the problem of the asymptotical stability of the high dimensional error dynamical networks is reduced to the same problem of a class of low dimensional error systems. The delay-dependent criteria guaranteeing global asymptotical stability for the error dynamical complex networks in terms of Liner Matrix Inequalities (LMIs) are derived based on free-weighting matrices technique and Lyapunov function. According to the convex characterization, a simple criterion is proposed. A numerical example is provided to demonstrate the effectiveness of the proposed results.
\end{abstract}

\section{Introduction}

Over the recent decades, dynamical complex networks are increasingly used to model a variety of phenomena of nature in power system, biological system, traffic system, and so on [1]. Many of these networks exhibit complexity in the overall topological properties and dynamical properties of the network nodes and the coupled units. The complex nature of the networks has resulted in a series of important research problems. In particular, one significant and interesting phenomenon is the synchronization of all its dynamics.

The pendulum-like system is a special kind of nonlinear system with periodic nonlinearity and multiple equilibria [2]. In practical engineering, there are many kinds of nonlinear pendulum-like system, such as phase-locked loops and various synchronous 
machines. With the development of the modern industry and control technique, all kinds of rotating electrical machines play more and more important roles in industry. Therefore, the pendulum-like system is worth being researched not only of academic significant, but also of practical value.

Recently, the coupled pendulum-like systems attract more and more researchers' attentions. Anticipating synchronization in a class of nonlinear dynamical systems is investigated in [3]. In [4], the global asymptotical stability and generalized synchronization of phase synchronous dynamical networks composed of multiinput multioutput pendulumlike systems via linear interconnections are investigated. Of particular note is that the global synchronization of the dynamical complex network composed of the pendulum-like systems is different from that of the general complex networks. The global synchronization of the dynamical complex networks composed of the pendulum-like systems is defined as phase synchronization introduced in [4]. But all of literatures above are not involving the coupling delays. However, time delay is unavoidably encountered, and it is the main cause of instability and poor performance of a system. Besides, the time-varying delays should be considered because they are more general than the constant cones. Thus, it is important and necessary to study the global synchronization of the dynamical complex networks composed of pendulum-like systems with time-varying coupling delays. In fact, the synchronization of the dynamical complex networks can be transformed into the global asymptotical stability of the corresponding error dynamical systems. In this paper, through studying the asymptotical stability of the corresponding error dynamical networks, several criteria guaranteeing the global phase synchronization of the dynamical network composed of multiinput multioutput pendulum-like systems with time-varying coupling delays are given. The effectiveness of the proved results is illustrated by a concrete example.

The rest of this paper is organized as follows. In Section 2, some preliminary results necessary for successive development are introduced. Section 3 contains our main results. In this section, we give some criteria guaranteeing the phase synchronization of the dynamical complex networks composed of the multiinput multioutput pendulum-like systems with time-varying coupling delays. The effectiveness of the proposed results is illustrated with a numerical example given in Section 4, and a brief conclusion is given in Section 5.

The following notions are used in this paper. $X^{T}$ indicate the transpose for real $X$. $X>0(X<0)$ means $X$ is a Hermitian and positive (negative) definite matrix. $I_{N}, I_{N n}$, $I_{N m}, I_{n}$, and $I_{m}$ are $N \times N, N n \times N n, N m \times N m, n \times n$, and $m \times m$ identity matrices, respectively. $I$ is an identity matrix with appropriate dimension. $\operatorname{diag}\left\{X_{1}, \ldots, X_{N}\right\}, U \otimes V$ are defined by

$$
\left(\begin{array}{ccc}
X_{1} & \cdots & 0 \\
\vdots & \ddots & \vdots \\
0 & \cdots & X_{n}
\end{array}\right), \quad U \otimes V=\left(\begin{array}{ccc}
u_{11} V & \cdots & u_{1 m} V \\
\vdots & \ddots & \vdots \\
u_{n 1} V & \cdots & u_{n m} V
\end{array}\right)
$$

If not explicitly stated, matrices are assumed to have compatible dimensions. 


\section{Preliminaries}

The nodes that compose a class of dynamical complex network can be described by following differential equation:

$$
\begin{aligned}
\dot{x}_{i} & =A x_{i}+B \varphi\left(\sigma_{i}\right), \\
\dot{\sigma}_{i} & =C x_{i}+D \varphi\left(\sigma_{i}\right),
\end{aligned}
$$

where variables $x_{i}=\left(x_{i 1}, x_{i 2}, \ldots, x_{i n}\right)^{T}$ and $\sigma_{i}=\left(\sigma_{i 1}, \sigma_{i 2}, \ldots, \sigma_{i m}\right)^{T}$ denote the state variables. $A \in \mathbb{R}^{n \times n}, B \in \mathbb{R}^{n \times m}, C \in \mathbb{R}^{m \times n}$, and $D \in \mathbb{R}^{m \times m}$ are constant matrices. The continuously differentiable vector function $\varphi\left(\sigma_{i}\right)=\left(\varphi_{1}\left(\sigma_{i 1}\right), \ldots, \varphi_{m}\left(\sigma_{i m}\right)\right)^{T}$ and $\varphi_{l}: \mathbb{R} \rightarrow \mathbb{R}$ is $\Delta_{l}$-periodic with finite number of zeros on the interval $\left[0, \Delta_{l}\right)(l=1, \ldots, m)$. The system equation (2.1) with $\Delta$-periodic $\sigma_{i}$ is called a pendulum-like system.

Proposition 2.1 (see [2]). If the solution $x_{i}(t)$ of the pendulum-like system (2.1) is bounded, then the functions $\varphi_{l}\left(\sigma_{i l}(t)\right)(l=1, \ldots, m)$, where $\sigma_{i l}(t)$ belongs to a solution of $(2.1)$, are uniformly continuous on $[0,+\infty]$.

The validity of this assertion follows from the facts that $\varphi_{l}\left(\sigma_{i l}(t)\right)$ is locally Lipschitz continuous and $\dot{\sigma}_{i l}(t)$ is bounded on $[0,+\infty)$.

Lemma 2.2 (see [2]). If $\alpha: \mathbb{R}_{+} \rightarrow \mathbb{R}$ belongs to $L^{2}[0,+\infty)$ and $\beta: \mathbb{R}_{+} \rightarrow \mathbb{R}$ belongs to $L^{2}[0,+\infty)$, then

$$
\tau(t)=\int_{0}^{t} \alpha(t-\tau) \beta(\tau) d \tau \longrightarrow 0, \quad t \longrightarrow+\infty
$$

Lemma 2.3 (see [2]). If $f: R_{+} \rightarrow R$ and is uniformly continuous and is $L^{2}[0,+\infty)$, then

$$
\lim _{t \rightarrow+\infty} f(t)=0
$$

\section{Main Results}

The dynamical complex network considered in this study is composed by $N$ identic pendulum-like nodes (2.1) with time-varying coupling delays, which could be described by the following equation:

$$
\begin{gathered}
\dot{x}_{i}(t)=A x_{i}(t)+B \varphi\left(\sigma_{i}(t)\right)+\sum_{j=1}^{N} G_{i j} \Gamma x_{j}(t-\tau(t)), \\
\dot{\sigma}_{i}(t)=C x_{i}(t)+D \varphi\left(\sigma_{i}(t)\right), \quad i=1,2, \ldots, N,
\end{gathered}
$$

where $\Gamma \in \mathbb{R}^{n \times n}$ defines the coupling between any two nodes. If node $j$ is linked node $i(i \neq j)$ directly, then $G_{i j}=G_{j i}=1$; otherwise, $G_{i j}=G_{j i}=0(i \neq j)$. The row sums of $G$ are zero, that is, $\sum_{j=1, j \neq i}^{n} G_{i j}=-G_{i i}(i=1, \ldots, N)$. The matrix $G=\left(G_{i j}\right) \in \mathbb{R}^{n \times n}$ indicates the connection 
topology and coupling strength, and $G$ is supposed to be irreducible. The time delay, $\tau(t)$, is a time-varying differentiable function that satisfies

$$
\begin{gathered}
0 \leq \tau(t) \leq h, \\
\dot{\tau}(t) \leq \mu,
\end{gathered}
$$

where $h>0$ and $\mu$ are constants.

Lemma 3.1 ( $\mathrm{Wu}[5])$. The eigenvalues of an irreducible matrix $G=\left(G_{i j}\right) \in \mathbb{R}^{N \times N}$ with $\sum_{j=1, j \neq i}^{N} G_{i j}=-G_{i i}(i=1, \ldots, N)$ satisfy the following properties.

(i) 0 is an eigenvalue of $G$ associated the eigenvector $(1,1, \ldots, 1)^{T}$.

(ii) If $G_{i j} \geq 0$ for $1 \leq i, j \leq N$, and $i \neq j$, then the real parts of all eigenvalues of $G$ are less than or equal to 0 , and all possible eigenvalues with zero part are the real eigenvalue 0 . In fact, 0 is an eigenvalue of $G$ with multiplicity 1.

There exists an orthogonal matrix $U$ satisfying $U U^{T}=I$ such that $U^{T} G U=\Lambda$, where $\Lambda$ is a diagonal matrix composed of eigenvalues of G. According to Lemma 3.1, it can be written as the following form:

$$
\Lambda=\operatorname{diag}(\lambda_{1}, \underbrace{\lambda_{2}, \ldots, \lambda_{2}}_{m_{2}}, \underbrace{\lambda_{3}, \ldots, \lambda_{3}}_{m_{3}}, \ldots, \underbrace{\lambda_{q}, \ldots, \lambda_{q}}_{m_{q}})
$$

where $\lambda_{1}=0$ is the maximum eigenvalue of multiply 1 , and $\lambda_{i}$ is the eigenvalue of multiply $m_{i}(i=2,3, \ldots, q)$ satisfying $m_{2}+\cdots+m_{q}=N-1$ and $0>\lambda_{2}>\lambda_{3}>\cdots>\lambda_{q}$.

Definition 3.2 (see [4]). The dynamical complex network model (3.1) is said to achieve global generalized phase synchronization if

$$
\begin{aligned}
& \lim _{t \rightarrow+\infty}\left\|x_{i}(t)-x_{s}(t)\right\|_{2}=0, \\
& \lim _{t \rightarrow+\infty}\left\|\sigma_{i}(t)-\sigma_{s}(t)\right\|_{2}=s,
\end{aligned}
$$

The sign $\|\cdot\|_{2}$ here means the Euclidean norm, and $s$ is a constant value. $\left(x_{s}(t), \sigma_{s}(t)\right)$ is the solution of each single node which can be equilibrium points, periodic orbits, or even nonperiodic orbits with

$$
\begin{aligned}
& \dot{x}_{s}=A x_{s}+B \varphi\left(\sigma_{s}\right) \\
& \dot{\sigma}_{s}=C x_{s}+D \varphi\left(\sigma_{s}\right) .
\end{aligned}
$$

From properties of the internal coupling matrix G given in Lemma 3.1, we know that $\sum_{j=1}^{N} G_{i j} \Gamma x_{s}(t-\tau(t))=0$, which could be added to the first equation in (3.5). By subtracting 
(3.5) from (3.1), we can get the following error dynamical system

$$
\begin{gathered}
\dot{e}_{1 i}(t)=A e_{1 i}(t)+B \phi\left(e_{2 i}(t), \sigma_{s}(t)\right)+\sum_{j=1}^{N} G_{i j} \Gamma e_{1 j}(t-\tau(t)), \quad i=1,2, \ldots, N, \\
\dot{e}_{2 i}(t)=C e_{1 i}(t)+D \phi\left(e_{2 i}(t), \sigma_{s}(t)\right),
\end{gathered}
$$

with $e_{1 i}(t)=x_{i}(t)-x_{s}(t), e_{2 i}(t)=\sigma_{i}(t)-\sigma_{s}(t)$, and $\phi\left(e_{2 i}(t), \sigma_{s}(t)\right)=\varphi\left(e_{2 i}(t)+\sigma_{s}(t)\right)-\varphi\left(\sigma_{s}(t)\right)$. Since $\varphi$ is a periodic function about $\sigma_{i}, \phi\left(e_{2 i}(t), \sigma_{s}(t)\right)$ also has a period of $\Delta$. According to the Kronecker product, system (3.6) could be written as follows:

$$
\begin{gathered}
\dot{e}_{1}(t)=\left(I_{N} \otimes A\right) e_{1}(t)+\left(I_{N} \otimes B\right) \Phi\left(e_{2}(t), \sigma(t)\right)+(G \otimes \Gamma) e_{1}(t-\tau(t)), \\
\dot{e}_{2}(t)=\left(I_{N} \otimes C\right) e_{1}(t)+\left(I_{N} \otimes D\right) \Phi\left(e_{2}(t), \sigma(t)\right),
\end{gathered}
$$

where

$$
\begin{gathered}
e_{1}=\left(e_{11}^{T}, \ldots, e_{1 N}^{T}\right)^{T}, \quad e_{2}=\left(e_{21}^{T}, \ldots, e_{2 N}^{T}\right)^{T}, \\
\Phi\left(e_{2}(t)\right)=\left(\phi^{T}\left(e_{21}(t), \sigma_{s}(t)\right), \ldots, \phi^{T}\left(e_{2 N}(t), \sigma_{s}(t)\right)\right)^{T} .
\end{gathered}
$$

Under such circumstances, system (3.7) could be regarded as a pendulum-like system with state delay. Thus, the synchronization problem of the dynamical network (3.1) can be transformed into the global asymptotical stability problem of the corresponding error dynamical system.

Theorem 3.3. Suppose that there exist scalars $h>0$ and $\mu$, matrices $P_{i}=P_{i}^{T}>0, Q_{i}=Q_{i}^{T}>0$, $R_{i}=R_{i}^{T}>0, \quad E_{i j}=E_{i j}^{T}>0(j=1,2), N_{i}=\left(N_{i 1}^{T} N_{i 2}^{T} N_{i 3}^{T} N_{i 4}^{T}\right)^{T}, S_{i}=\left(S_{i 1}^{T} S_{i 2}^{T} S_{i 3}^{T} S_{i 4}^{T}\right)^{T}$, $M_{i}=\left(M_{i 1}^{T} M_{i 2}^{T} M_{i 3}^{T} M_{i 4}^{T}\right)^{T}$ and diagonal matrices $\kappa_{i}, \delta_{i}, \varepsilon_{i}$ with $\delta_{i}>0, \varepsilon_{i}>0$ such that the following inequalities are satisfied:

$$
\Pi=\left(\begin{array}{ccccccccc}
\Pi_{11} & \Pi_{12} & \Pi_{13} & \Pi_{14} & C^{T} \varepsilon_{i} & h N_{i 1} & h S_{i 1} & h M_{i 1} & h A^{T}\left(E_{i 1}+E_{i 2}\right) \\
* & \Pi_{22} & \Pi_{23} & -N_{i 4}^{T}+S_{i 4}^{T} & 0 & h N_{i 2} & h S_{i 2} & h M_{i 2} & h \lambda_{i} \Gamma^{T}\left(E_{i 1}+E_{i 2}\right) \\
* & * & \Pi_{33} & -S_{i 4}^{T}-M_{i 4}^{T} & 0 & h N_{i 3} & h S_{i 3} & h M_{i 3} & 0 \\
* & * & * & \Pi_{44} & D^{T} \varepsilon_{i} & 0 & 0 & 0 & h B^{T}\left(E_{i 1}+E_{i 2}\right) \\
* & * & * & * & -\varepsilon_{i} & 0 & 0 & 0 & 0 \\
* & * & * & * & * & -h E_{i 1} & 0 & 0 & 0 \\
* & * & * & * & * & * & -h E_{i 1} & 0 & 0 \\
* & * & * & * & * & * & * & -h E_{i 2} & 0 \\
* & * & * & * & * & * & * & * & -h\left(E_{i 1}+E_{i 2}\right)
\end{array}\right)<0,
$$


where $\Pi_{11}=P_{i} A+A^{T} P_{i}+Q_{i}+R_{i}+N_{i 1}+N_{i 1}^{T}+M_{i 1}+M_{i 1}^{T}, \Pi_{12}=P_{i} \lambda_{i} \Gamma-N_{i 1}+N_{i 2}^{T}+S_{i 1}+M_{i 2}^{T}$ $\Pi_{13}=N_{i 3}^{T}-M_{i 1}+M_{i 3}^{T}-S_{i 1}, \Pi_{14}=P_{i} B+N_{i 4}^{T}+M_{i 4}^{T}+1 / 2 C^{T} \kappa_{i}, \Pi_{22}=-(1-\mu) Q_{i}-N_{i 2}-N_{i 2}^{T}+S_{i 2}+S_{i 2}^{T}$ $\Pi_{23}=-S_{i 2}+S_{i 3}^{T}-M_{i 2}-N_{i 3}^{T}, \Pi_{33}=-R_{i}-S_{i 3}-S_{i 3}^{T}-M_{i 3}-M_{i 3}^{T}, \Pi_{44}=\delta_{i}+1 / 2 \kappa_{i} D+(1 / 2) D^{T} \kappa_{i}$, $v_{i}=\operatorname{diag}\left(v_{i 1}, \ldots, v_{i m}\right)$ with

$$
v_{i l}=\frac{\int_{0}^{\Delta_{l}} \int_{0}^{\Delta_{l}} \phi_{l}\left(y_{i l}(t), \sigma_{s l}(t)\right) d y_{i l} d \sigma_{s l}}{\int_{0}^{\Delta_{l}} \int_{0}^{\Delta_{l}}\left|\phi_{l}\left(y_{i l}(t), \sigma_{s l}(t)\right)\right| d y_{i l} d \sigma_{s l}} \quad l=1,2, \ldots, m,
$$

where $y(t)=\left(U^{T} \otimes I_{m}\right) e_{2}(t)=\left(y_{1}^{T}(t), \ldots, y_{N}^{T}(t)\right)^{T}$, and $U$ is a selected orthogonal matrix satisfying $U^{T} G U=\Lambda$, where $\Lambda$ is defined as (3.3). Then the delayed pendulum-like system (3.7) with time-varying coupling delay $\tau(t)$ satisfying (3.2) is global asymptotic, stable and the corresponding dynamical network (3.1) achieves phase synchronization.

Proof. Recall the property of Kronecker product [6]

$$
(M \otimes N)(G \otimes D)=(M G) \otimes(N D),
$$

where $M \in \mathbb{R}^{k \times m}, N \in \mathbb{R}^{p \times s}, G \in \mathbb{R}^{m \times n}$, and $D \in \mathbb{R}^{s \times q}$. Choose an orthogonal matrix $U$ satisfying $U^{T} G U=\Lambda$, where $\Lambda$ is defined as the form of (3.3). Let

$$
\begin{aligned}
z(t) & =\left(U^{T} \otimes I_{n}\right) e_{1}(t)=\left(z_{1}^{T}(t), \ldots, z_{N}^{T}(t)\right)^{T}, \\
y(t) & =\left(U^{T} \otimes I_{m}\right) e_{2}(t)=\left(y_{1}^{T}(t), \ldots, y_{N}^{T}(t)\right)^{T} .
\end{aligned}
$$

Premultiplying two formulas of (3.7) by $U^{T} \otimes I_{n}$ and $U^{T} \otimes I_{m}$, respectively, yields

$$
\begin{gathered}
\dot{z}(t)=\left(I_{N} \otimes A\right) z(t)+\left(I_{N} \otimes B\right) \Phi\left(y(t), \sigma_{S}(t)\right)+(\Lambda \otimes \Gamma) z(t-\tau(t)), \\
\dot{y}(t)=\left(I_{N} \otimes C\right) z(t)+\left(I_{N} \otimes D\right) \Phi\left(y(t), \sigma_{S}(t)\right),
\end{gathered}
$$

yielding

$$
\begin{aligned}
& \dot{z}_{i}(t)=A z_{i}(t)+\lambda_{i} \Gamma z_{i}(t-\tau(t))+B \phi\left(y_{i}(t), \sigma_{s}(t)\right), \\
& \dot{y}_{i}(t)=C z_{i}(t)+D \phi\left(y_{i}(t), \sigma_{s}(t)\right), \quad i=1,2, \ldots, N .
\end{aligned}
$$

Introduce the new functions

$$
F_{l}\left(y_{i l}(t)\right)=\phi_{l}\left(y_{i l}(t), \sigma_{s l}(t)\right)-v_{i l}\left|\phi_{l}\left(y_{i l}(t), \sigma_{s l}(t)\right)\right|,
$$

therefore,

$$
\int_{0}^{\Delta_{l}} F_{l}\left(y_{i l}\right) d y_{i l}=0
$$


Journal of Inequalities and Applications

and the function $F_{l}$ has a mean value zero. We consider the following Lyapunov function:

$$
V=V_{1}+\sum_{k=1}^{m} \kappa_{i k} \int_{0}^{y_{i k}} F_{k}(\tau) d \tau
$$

where

$$
\begin{aligned}
V_{1}= & z_{i}^{T}(t) P_{i} z_{i}(t)+\int_{t-\tau(t)}^{t} z_{i}^{T}(\alpha) Q_{i} z_{i}(\alpha) d \alpha+\int_{t-h}^{t} z_{i}^{T}(\alpha) R_{i} z_{i}(\alpha) d \alpha \\
& +\int_{-h}^{0} \int_{t+\theta}^{t} \dot{z}_{i}^{T}(\alpha)\left(E_{i 1}+E_{i 2}\right) \dot{z}_{i}(\alpha) d \alpha d \theta
\end{aligned}
$$

By the New-Leibniz formula, we have

$$
\int_{t-h}^{t} \dot{z}_{i}(\alpha) d \alpha=z_{i}(t)-z_{i}(t-h)
$$

Then, in virtue of (3.20), we have the following formulations for any matrices $N_{i}, S_{i}, M_{i}$ with appropriate dimensions:

$$
\begin{aligned}
\Phi_{1}= & {\left[z_{i}^{T}(t) N_{i 1}+z_{i}^{T}(t-\tau(t)) N_{i 2}+z_{i}^{T}(t-h) N_{i 3}+\phi^{T}\left(y_{i}(t), \sigma_{s}(t)\right) N_{i 4}\right]\left[z_{i}(t)-z_{i}(t-\tau(t))\right.} \\
& \left.-\int_{t-\tau(t)}^{t} \dot{z}_{i}(\alpha) d \alpha\right]=0 \\
\Phi_{2}= & {\left[z_{i}^{T}(t) S_{i 1}+z_{i}^{T}(t-\tau(t)) S_{i 2}+z_{i}^{T}(t-h) S_{i 3}+\phi^{T}\left(y_{\mathrm{i}}(t), \sigma_{s}(t)\right) S_{i 4}\right]\left[z_{i}(t-\tau(t))-z_{i}(t-h)\right.} \\
& \left.-\int_{t-h}^{t-\tau(t)} \dot{z}_{i}(\alpha) d \alpha\right]=0, \\
\Phi_{3}= & {\left[z_{i}^{T}(t) M_{i 1}+z_{i}^{T}(t-\tau(t)) M_{i 2}+z_{i}^{T}(t-h) M_{i 3}+\phi^{T}\left(y_{i}(t), \sigma_{s}(t)\right) M_{i 4}\right]\left[z_{i}(t)-z_{i}(t-h)\right.} \\
& \left.-\int_{t-h}^{t} \dot{z}_{i}(\alpha) d \alpha\right]=0 .
\end{aligned}
$$


Calculating the derivative of $V_{1}$ along the solutions of (3.15) and adding $2 \Phi_{1}$ from (3.21), $2 \Phi_{2}$ from (3.22), and $2 \Phi_{3}$ from (3.23) to it, we have

$$
\begin{aligned}
& \dot{V}_{1}=2 z_{i}^{T}(t) P_{i}\left[A z_{i}(t)+\lambda_{i} \Gamma z_{i}(t-\tau(t))+B \phi\left(y_{i}(t), \sigma_{s}(t)\right)\right]+z_{i}^{T}(t) Q_{i} z_{i}(t) \\
& -(1-\dot{\tau}(t)) z_{i}^{T}(t-\tau(t)) Q_{i} z_{i}(t-\tau(t))+z_{i}^{T}(t) R_{i} z_{i}(t)-z_{i}^{T}(t-h) R_{i} z_{i}(t-h) \\
& +h \dot{z}_{i}^{T}(t)\left(E_{i 1}+E_{i 2}\right) \dot{z}_{i}(t)-\int_{t-h}^{t} \dot{z}_{i}^{T}(\alpha)\left(E_{i 1}+E_{i 2}\right) \dot{z}_{i}(\alpha) d \alpha+2 \Phi_{1}+2 \Phi_{2}+2 \Phi_{3} \\
& \leq 2 z_{i}^{T}(t) P_{i}\left[A z_{i}(t)+\lambda_{i} \Gamma z_{i}(t-\tau(t))+B \phi\left(y_{i}(t), \sigma_{S}(t)\right)\right]+z_{i}^{T}(t) Q_{i} x_{i}(t) \\
& -(1-\mu) z_{i}^{T}(t-\tau(t)) Q_{i} z_{i}(t-\tau(t))+z_{i}^{T}(t) R_{i} x(t)-z_{i}^{T}(t-h) R_{i} z_{i}(t-h) \\
& +h \dot{z}_{i}^{T}(t)\left(E_{i 1}+E_{i 2}\right) \dot{z}_{i}(t)-\int_{t-h}^{t} \dot{z}_{i}^{T}(\alpha)\left(E_{i 1}+E_{i 2}\right) \dot{z}_{i}(\alpha) d \alpha+2 \Phi_{1}+2 \Phi_{2}+2 \Phi_{3} \\
& =\zeta^{T}(t) \Lambda_{1} \zeta(t)+h \dot{z}_{i}^{T}(t)\left(E_{i 1}+E_{i 2}\right) \dot{z}_{i}(t)-\int_{t-h}^{t} \dot{z}_{i}^{T}(\alpha)\left(E_{i 1}+E_{i 2}\right) \dot{z}_{i}(\alpha) d \alpha \\
& -2 \zeta^{T}(t) N_{i} \int_{t-\tau(t)}^{t} \dot{z}_{i}(\alpha) d \alpha-2 \zeta^{T}(t) S_{i} \int_{t-h}^{t-\tau(t)} \dot{z}_{i}(\alpha) d \alpha-2 \zeta^{T}(t) M_{i} \int_{t-h}^{t} \dot{z}_{i}(\alpha) d \alpha \\
& =\zeta^{T}(t)\left(\Lambda_{1}+h A_{k}^{T}\left(E_{i 1}+E_{i 2}\right) A_{k}+\tau(t) N_{i} E_{i 1}^{-1} N_{i}^{T}+(h-\tau(t)) S_{i} E_{i 1}^{-1} S_{i}^{T}+h M_{i} E_{i 2}^{-1} M_{i}^{T}\right) \zeta(t) \\
& -\int_{t-\tau(t)}^{t}\left[\zeta^{T}(t) N_{i}+\dot{z}_{i}^{T}(\alpha) E_{i 1}\right] E_{i 1}^{-1}\left[N_{i}^{T} \zeta(t)+E_{i 1} \dot{z}_{i}(\alpha)\right] d \alpha \\
& -\int_{t-h}^{t-\tau(t)}\left[\zeta^{T}(t) S_{i}+\dot{z}_{i}^{T}(\alpha) E_{i 1}\right] E_{i 1}^{-1}\left[S_{i}^{T} \zeta(t)+E_{i 1} \dot{z}_{i}(\alpha)\right] d \alpha \\
& -\int_{t-h}^{t}\left[\zeta^{T}(t) M_{i}+\dot{z}_{i}^{T}(\alpha) E_{i 2}\right] E_{i 2}^{-1}\left[M_{i}^{T} \zeta(t)+E_{i 2} \dot{z}_{i}(\alpha)\right] d \alpha \\
& \leq \zeta^{T}(t)\left(\Lambda_{1}+h A_{k}^{T}\left(E_{i 1}+E_{i 2}\right) A_{k}+h N_{i} E_{i 1}^{-1} N_{i}^{T}+h S_{i} E_{i 1}^{-1} S_{i}^{T}+h M_{i} E_{i 2}^{-1} M_{i}^{T}\right) \zeta(t) \\
& -\int_{t-\tau(t)}^{t}\left[\zeta^{T}(t) N_{i}+\dot{z}_{i}^{T}(\alpha) E_{i 1}\right] E_{i 1}^{-1}\left[N_{i}^{T} \zeta(t)+E_{i 1} \dot{z}_{i}(\alpha)\right] d \alpha \\
& -\int_{t-h}^{t-\tau(t)}\left[\zeta^{T}(t) S_{i}+\dot{z}_{i}^{T}(\alpha) E_{i 1}\right] E_{i 1}^{-1}\left[S_{i}^{T} \zeta(t)+E_{i 1} \dot{z}_{i}(\alpha)\right] d \alpha \\
& -\int_{t-h}^{t}\left[\zeta^{T}(t) M_{i}+\dot{z}_{i}^{T}(\alpha) E_{i 2}\right] E_{i 2}^{-1}\left[M_{i}^{T} \zeta(t)+E_{i 2} \dot{z}_{i}(\alpha)\right] d \alpha,
\end{aligned}
$$


where

$$
\begin{aligned}
& \zeta^{T}(t)=\left[\begin{array}{llll}
z_{i}^{T}(t) & z_{i}^{T}(t-\tau(t)) & z_{i}^{T}(t-h) & \phi^{T}\left(y_{i}(t), \sigma_{s}(t)\right)
\end{array}\right], \\
& \Lambda_{1}=\left(\begin{array}{cccc}
\Lambda_{11} & \Lambda_{12} & N_{i 3}^{T}-M_{i 1}+M_{i 3}^{T}-S_{i 1} & P_{i} B+N_{i 4}^{T}+M_{i 4}^{T} \\
* & \Lambda_{22} & -S_{i 2}+S_{i 3}^{T}-M_{i 2}-N_{i 3}^{T} & -N_{i 4}^{T}+S_{i 4}^{T} \\
* & * & -R_{i}-S_{i 3}-S_{i 3}^{T}-M_{i 3}-M_{i 3}^{T} & -S_{i 4}^{T}-M_{i 4}^{T} \\
* & * & * & 0
\end{array}\right), \\
& \Lambda_{11}=P_{i} A+A^{T} P_{i}+Q_{i}+R_{i}+N_{i 1}+N_{i 1}^{T}+M_{i 1}+M_{i 1}^{T} \text {, } \\
& \Lambda_{12}=P_{i} \lambda_{i} \Gamma-N_{i 1}+N_{i 2}^{T}+S_{i 1}+M_{i 2}^{T} \text {, } \\
& \Lambda_{22}=-(1-\mu) Q_{i}-N_{i 2}-N_{i 2}^{T}+S_{i 2}+S_{i 2}^{T} \text {, } \\
& A_{k}=\left[\begin{array}{llll}
A & \lambda_{i} \Gamma & 0 & B
\end{array}\right] .
\end{aligned}
$$

Since $E_{i l}>0, l=1,2$, then the last three parts are all less than 0 . So if $\Lambda_{1}+h A_{k}^{T}\left(E_{i 1}+E_{i 2}\right) A_{k}+$ $h N_{i} E_{i 1}^{-1} N_{i}^{T}+h S_{i} E_{i 1}^{-1} S_{i}^{T}+h M_{i} E_{i 2}^{-1} M_{i}^{T}<0$, then $\dot{V}_{1}<0$.

Then,

$$
\begin{gathered}
\dot{V}=\dot{V}_{1}+\sum_{k=1}^{m} \kappa_{i k} F_{k}\left(y_{i k}(t)\right) \dot{y}_{i k}(t) \\
=\dot{V}_{1}+\sum_{k=1}^{m}\left[\kappa_{i k} \phi_{k}\left(y_{i k}(t), \sigma_{s k}(t)\right) \dot{y}_{i k}(t)-\kappa_{i k} v_{i k}\left|\phi_{k}\left(y_{i k}(t), \sigma_{s k}(t)\right)\right| \dot{y}_{i k}(t)-\varepsilon_{i k} \dot{y}_{i k}^{2}(t)\right. \\
\left.\quad-\delta_{i k} \phi_{k}^{2}\left(y_{i k}(t), \sigma_{s k}(t)\right)+\varepsilon_{i k} \dot{y}_{i k}^{2}(t)+\delta_{i k} \phi_{k}^{2}\left(y_{i k}(t), \sigma_{s k}(t)\right)\right] .
\end{gathered}
$$

In virtue of condition (3.10) of the theorem, there exist $\delta_{i 0 k}>0$ and $\varepsilon_{i 0 k}>0$ such that

$$
\begin{gathered}
\kappa_{i k} \nu_{i k}\left|\phi_{k}\left(y_{i k}(t), \sigma_{s k}(t)\right)\right| \dot{y}_{i k}(t)+\varepsilon_{i k} \dot{y}_{i k}^{2}(t)+\delta_{i k} \phi_{k}^{2}\left(y_{i k}(t), \sigma_{s k}(t)\right) \\
\geq \varepsilon_{i 0 k} \dot{y}_{i k}^{2}(t)+\delta_{i 0 k} \phi_{k}^{2}\left(y_{i k}(t), \sigma_{s k}(t)\right) .
\end{gathered}
$$

Thus, the following inequality is satisfied:

$$
\begin{aligned}
\dot{V}(t) & +\sum_{k=1}^{m}\left[\varepsilon_{i 0 k} y_{i k}^{2}(t)+\delta_{i 0 k} \phi_{k}^{2}\left(y_{i k}(t), \sigma_{s k}(t)\right)\right] \\
& \leq \dot{V}_{1}(t)+\sum_{k=1}^{m}\left[\kappa_{i k} \phi_{k}\left(y_{i k}(t), \sigma_{s k}(t)\right) \dot{y}_{i k}(t)+\varepsilon_{i k} y_{i k}^{2}(t)+\delta_{i k} \phi_{i k}^{2}\left(y_{i k}(t), \sigma_{s k}(t)\right)\right] .
\end{aligned}
$$


Assuming that

$$
\Upsilon(t)=\sum_{k=1}^{m}\left[\kappa_{i k} \phi_{k}\left(y_{i k}(t), \sigma_{s k}(t)\right) \dot{y}_{i k}(t)+\varepsilon_{i k} \sigma_{i k}^{2}(t)+\delta_{i k} \phi_{k}^{2}\left(y_{i k}(t), \sigma_{s k}(t)\right)\right] .
$$

substituting the second equation of (3.15) into (3.29), we have

$$
\dot{V}_{1}(t)+\Upsilon(t) \leq \zeta^{T}(t)\left(\Lambda_{1}+h A_{k}^{T}\left(E_{i 1}+E_{i 2}\right) A_{k}+h N_{i} E_{i 1}^{-1} N_{i}^{T}+h S_{i} E_{i 1}^{-1} S_{i}^{T}+h M_{i} E_{i 2}^{-1} M_{i}^{T}+\Lambda_{2}\right) \zeta(t),
$$

where

$$
\Lambda_{2}=\left(\begin{array}{cccc}
C^{T} \varepsilon_{i} C & 0 & 0 & \frac{1}{2} C^{T} \kappa_{i}+C^{T} \varepsilon_{i} D \\
* & 0 & 0 & 0 \\
* & * & 0 & 0 \\
* & * & * & \frac{1}{2} \kappa_{i} D+\frac{1}{2} D^{T} \kappa_{i}+D^{T} \varepsilon_{i} D+\delta_{i}
\end{array}\right),
$$

and $\Lambda_{1}+h A_{k}^{T}\left(E_{i 1}+E_{i 2}\right) A_{k}+h N_{i} E_{i 1}^{-1} N_{i}^{T}+h S_{i} E_{i 1}^{-1} S_{i}^{T}+h M_{i} E_{i 2}^{-1} M_{i}^{T}+\Lambda_{2}$ is equivalent to $\Pi$ in (3.9) by Schur complements. The inequality condition (3.9) of the theorem guarantees that

$$
\zeta^{T}(t)\left(\Lambda_{1}+h A_{k}^{T}\left(E_{i 1}+E_{i 2}\right) A_{k}+h N_{i} E_{i 1}^{-1} N_{i}^{T}+h S_{i} E_{i 1}^{-1} S_{i}^{T}+h M_{i} E_{i 2}^{-1} M_{i}^{T}+\Lambda_{2}\right) \zeta(t)<0 .
$$

Then, there exists a diagonal matrix $\rho_{i}=\operatorname{diag}\left(\rho_{i 1}, \rho_{i 2}, \ldots, \rho_{i n}\right), \rho_{i k}>0, k=1,2, \ldots, n$

$$
\zeta^{T}(t)\left(\Lambda_{1}+h A_{k}^{T}\left(E_{i 1}+E_{i 2}\right) A_{k}+h N_{i} E_{i 1}^{-1} N_{i}^{T}+h S_{i} E_{i 1}^{-1} S_{i}^{T}+h M_{i} E_{i 2}^{-1} M_{i}^{T}+\Lambda_{2}\right) \zeta(t)<-\sum_{k=1}^{m} \rho_{i k} z_{i k^{\prime}}^{2}
$$

namely,

$$
\dot{V}(z(t), \sigma(t))+\sum_{k=1}^{m}\left[\varepsilon_{i 0 k} \dot{y}_{i k}^{2}(t)+\delta_{i 0 k} \phi_{k}^{2}\left(y_{i k}(t), \sigma_{s k}(t)\right)\right]<-\sum_{k=1}^{m} \rho_{i k} z_{i k}^{2} .
$$

Hence,

$$
V(t)-V(0) \leq-\sum_{k=1}^{m} \int_{0}^{t}\left[\varepsilon_{i 0 k} \dot{y}_{i k}^{2}(t)+\delta_{i 0 k} \phi_{k}^{2}\left(y_{i k}(t), \sigma_{s k}(t)\right)\right] d t-\sum_{k=1}^{n} \int_{0}^{t} \rho_{i k} z_{i k}^{2}(t) d t,
$$


for all $t \geq 0$. The function $V(t)$ is bounded because solutions $z_{k}(t)$ are bounded, and the functions $F_{k}(\tau)$ have mean value zero. Therefore, from (3.35), we have

$$
\begin{gathered}
\int_{0}^{+\infty} \phi_{k}^{2}\left(y_{i k}(t), \sigma_{s k}(t)\right) d t<+\infty, \\
\int_{0}^{+\infty} \dot{y}_{i k}^{2}(t) d t<+\infty \\
\int_{0}^{+\infty} z_{i k}^{2}(t) d t<+\infty
\end{gathered}
$$

From Proposition 2.1, it follows that the functions $\phi\left(y_{i}(t), \sigma_{s}(t)\right)$ are uniformly continuous on $[0,+\infty)$. And from (3.36) and Lemma 3.1, functions $\phi\left(y_{i}(t), \sigma_{s}(t)\right)$ tend to zero as $t \rightarrow+\infty$,

$$
\lim _{t \rightarrow+\infty} \phi_{k}^{2}\left(y_{i k}(t), \sigma_{s k}(t)\right)=0
$$

Further, we have

$$
y_{i k}(t) \longrightarrow \hat{y}_{i k}(t), \quad t \longrightarrow+\infty,
$$

where $\phi_{k}\left(\hat{y}_{i k}(t), \sigma_{s k}(t)\right)=0(k=1,2, \ldots, m)$. Let us now consider the the first equation of system (3.15). We can represent $z_{i}(t)$ in the form

$$
z_{i}(t)=e^{A t} z_{i}(0)+\int_{0}^{t} e^{A(t-s)} \lambda_{i} \Gamma z_{i}(s-\tau(s)) d s+\int_{0}^{t} e^{A(t-s)} B \phi_{i k}^{2}\left(y_{i k}(s), \sigma_{s}(t)\right) d s .
$$

From (3.36), (3.38), and Lemma 2.3, we have

$$
\begin{gathered}
\lim _{t \rightarrow+\infty} \int_{0}^{t} e^{A(t-s)} B \phi_{i k}\left(y_{i k}(s), \sigma_{s}(s)\right) d s=0 \\
\lim _{t \rightarrow+\infty} \int_{0}^{t} e^{A(t-s)} \lambda_{i} \Gamma z_{i}(s-\tau(s)) d s=0
\end{gathered}
$$

Furthermore, since $A$ is Hurwitzian, the following conclusion is obtained:

$$
\lim _{t \rightarrow+\infty} z_{i}(t)=0
$$

The conditions (3.40) and (3.43) show that every solution $\left(z_{i}(t), y_{i}(t)\right)$ of the pendulum-like system (3.15) converges to a certain equilibrium $\left(z_{i e q}=0,\left(y_{i e q}\right)_{l}=\widehat{y}_{i l}\right)$ with $\phi_{i l}\left(\widehat{y}_{i l}(t), \sigma_{s l}(t)\right)=$ $0(l=1,2, \ldots, m)$. Namely, the pendulum-like system (3.7) is global asymptotic stable.

Remark 3.4. It is shown from the formula (3.3) that the coupling matrix $G$ has $q$ different eigenvalues. Therefore, it is just needed to examine $q$ LMIs groups in (3.9) and (3.10). In 
addition, according to the convex properties of LMI [7], $q-3$ groups of LMIs corresponding to $\lambda_{3}, \ldots, \lambda_{q-1}$ can be written as a linear combination of the tow groups of LMIs with the second-maximum $\lambda_{2}$ and the minimum eigenvalue $\lambda_{q}$. Therefore, above criterion only needs to examine three groups of LMIs corresponding to the largest, second largest, and the smallest distinct eigenvalues of $G$, respectively. Furthermore, note that the system (3.1) with $\lambda_{1}=0$ just corresponds to the synchronous manifold, which is not required to be verified. Hence, if (3.9) and (3.10) hold for $q=2$ and $N$, the nonlinear pendulum-like dynamical network will achieve phase synchronization.

Corollary 3.5. Suppose that there exist scalars $h>0$ and $\mu$, matrices $P_{i}=P_{i}^{T}>0, Q_{i}=Q_{i}^{T}>0$, $R_{i}=R_{i}^{T}>0, E_{i j}=E_{i j}^{T}>0(j=1,2), N_{i}=\left(N_{i 1}^{T} N_{i 2}^{T} N_{i 3}^{T} N_{i 4}^{T}\right)^{T}, S_{i}=\left(S_{i 1}^{T} S_{i 2}^{T} S_{i 3}^{T} S_{i 4}^{T}\right)^{T}$, $M_{i}=\left(M_{i 1}^{T} M_{i 2}^{T} M_{i 3}^{T} M_{i 4}^{T}\right)^{T}$ and diagonal matrices $\kappa_{i}, \delta_{i}, \varepsilon_{i}$ with $\delta_{i}>0, \varepsilon_{i}>0$, such that the following inequalities are satisfied:

$$
\left(\begin{array}{ccccccccc}
\Pi_{11} & \Pi_{12} & \Pi_{13} & \Pi_{14} & C^{T} \varepsilon_{i} & h N_{i 1} & h S_{i 1} & h M_{i 1} & h A^{T}\left(E_{i 1}+E_{i 2}\right) \\
* & \Pi_{22} & \Pi_{23} & -N_{i 4}^{T}+S_{i 4}^{T} & 0 & h N_{i 2} & h S_{i 2} & h M_{i 2} & h \lambda_{i} \Gamma^{T}\left(E_{i 1}+E_{i 2}\right) \\
* & * & \Pi_{33} & -S_{i 4}^{T}-M_{i 4}^{T} & 0 & h N_{i 3} & h S_{i 3} & h M_{i 3} & 0 \\
* & * & * & \Pi_{44} & D^{T} \varepsilon_{i} & 0 & 0 & 0 & h B^{T}\left(E_{i 1}+E_{i 2}\right) \\
* & * & * & * & -\varepsilon_{i} & 0 & 0 & 0 & 0 \\
* & * & * & * & * & -h E_{i 1} & 0 & 0 & 0 \\
* & * & * & * & * & * & -h E_{i 1} & 0 & 0 \\
* & * & * & * & * & * & * & -h E_{i 2} & 0 \\
* & * & * & * & * & * & * & * & -h\left(E_{i 1}+E_{i 2}\right)
\end{array}\right)<0
$$

where $\Pi_{11}=P_{i} A+A^{T} P_{i}+Q_{i}+R_{i}+N_{i 1}+N_{i 1}^{T}+M_{i 1}+M_{i 1}^{T}, \Pi_{12}=\lambda_{i} P_{i} \Gamma-N_{i 1}+N_{i 2}^{T}+S_{i 1}+M_{i 2}^{T}$, $\Pi_{13}=N_{i 3}^{T}-M_{i 1}+M_{i 3}^{T}-S_{i 1}, \Pi_{14}=P_{i} B+N_{i 4}^{T}+M_{i 4}^{T}+(1 / 2) C^{T} \kappa_{i}, \Pi_{22}=-(1-\mu) Q_{i}-N_{i 2}-N_{i 2}^{T}+S_{i 2}+S_{i 2}^{T}$ $\Pi_{23}=-S_{i 2}+S_{i 3}^{T}-M_{i 2}-N_{i 3}^{T}, \Pi_{33}=-R_{i}-S_{i 3}-S_{i 3}^{T}-M_{i 3}-M_{i 3}^{T}, \Pi_{44}=\delta_{i}+(1 / 2) \kappa_{i} D+(1 / 2) D^{T} \kappa_{i}$, and $v_{i}$ defined as the Theorem 3.3. Then, the dynamical network (3.1) with time-varying coupling delay $\tau(t)$ satisfying (3.2) achieves phase synchronization.

\section{Numerical Example}

The example given in this section is based on concrete systems studied in the theory of interconnected phase-locked loops (PLLs), which are frequently observed in electrical and engineering aspects. PLL could be treated as a representative for pendulum-like system, where model is described by (2.1) after certain simplifications [8]. 


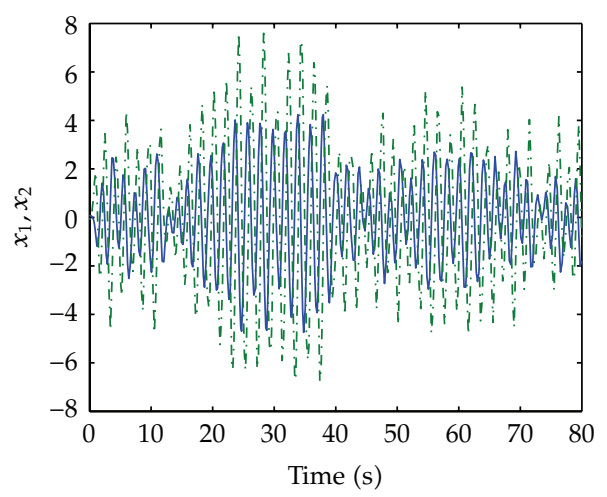

(a)

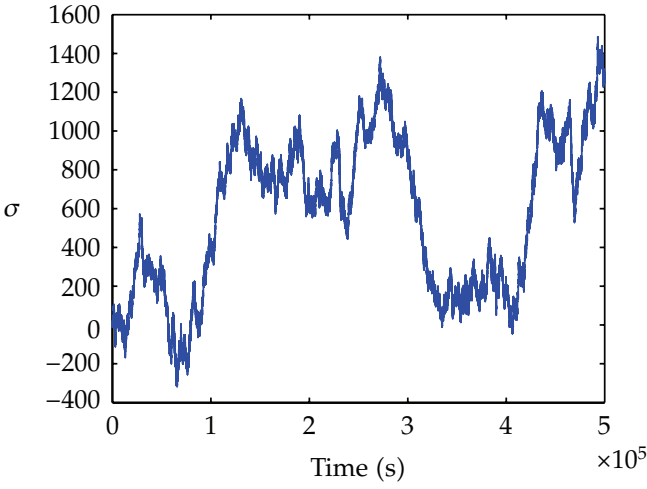

(b)

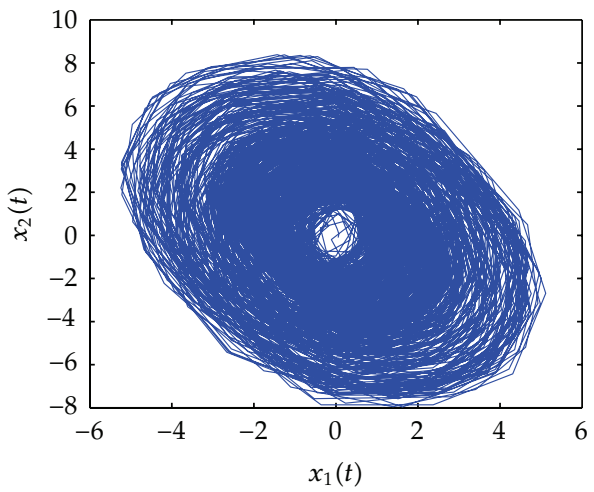

(c)

Figure 1: Simulation of each PLL node: $x_{1}, x_{2}$, and $\sigma$ and the phase plot between $x_{1}, x_{2}$, and $\sigma$.

The dynamical complex network (3.1) is composed of third-order PLL nodes with the following parameters:

$$
A=\left[\begin{array}{cc}
-1 & -2 \\
5 & 1
\end{array}\right], \quad B=\left[\begin{array}{l}
1 \\
4
\end{array}\right], \quad C=\left[\begin{array}{ll}
-2 & 2
\end{array}\right], \quad D=3
$$

and the nonlinear function $\varphi\left(\sigma_{i}\right)=\sin \left(\sigma_{i}\right)$. The network topology parameters in (4.1) are picked as

$$
G=\left[\begin{array}{ccccc}
-2 & 1 & 0 & 0 & 1 \\
1 & -2 & 1 & 0 & 0 \\
0 & 1 & -2 & 1 & 0 \\
0 & 1 & 1 & -3 & 1 \\
1 & 0 & 0 & 1 & -2
\end{array}\right]
$$




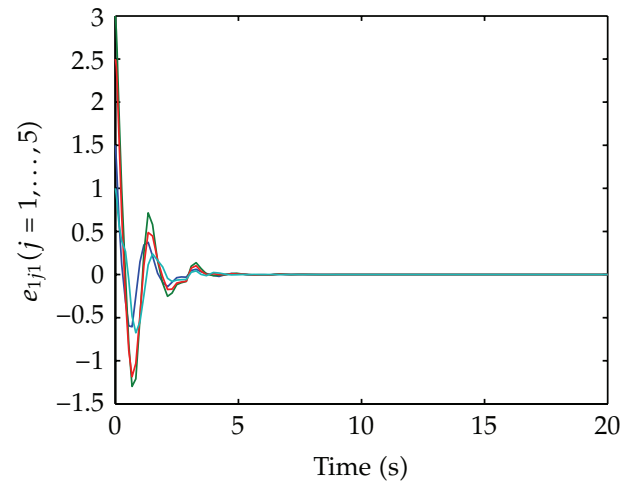

(a)

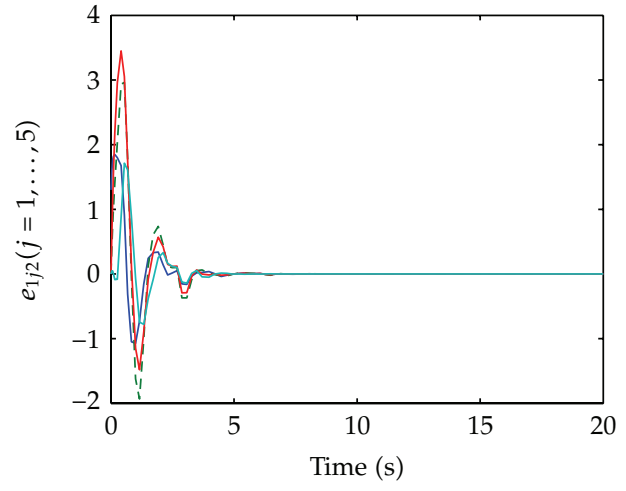

(b)

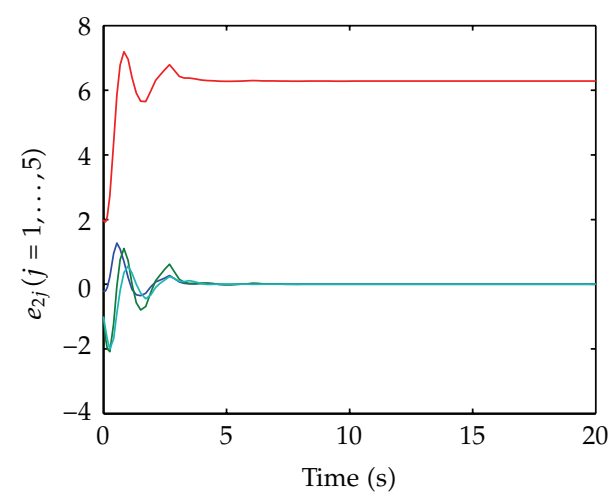

(c)

Figure 2: The error variations in (4.1): $e_{1 j 1}=x_{11}-x_{j 1} ; e_{1 j 2}=x_{12}-x_{j 2} ; e_{2 j}=\sigma_{1}-\sigma_{j}$, and $j=2, \ldots, 5$.

Assume that the inner-coupling matrix is $\Gamma=\operatorname{diag}\{1,1,1,1,1\}$. Eigenvalues of the coupling matrix $G$ can be calculated as

$$
\lambda_{1}=0, \quad \lambda_{2}=-1.382, \quad \lambda_{3}=-2, \quad \lambda_{4}=-3.618, \quad \lambda_{5}=-4 .
$$

The chaotic phenomenon of the state variables $x_{1}$ and $x_{2}$ of the single PLL node is shown in Figure 1. It is also observed that the phase variable $\sigma$ is unbounded, so there is no chaotic phenomenon about $\sigma$ in plane phase space. However, chaotic phenomenon appears on the cylindrical surface of cylindrical phase space. This peculiar phenomenon to pendulum-like system is called the chaos on cylindrical surface $[9,10]$. Although the global asymptotical stability of pendulum-like system network model (4.1) may not be ensured, the global phase synchronization could be achieved. According to Corollary 3.5, when $h=0.2, \mu=0.1$, the LMIs (3.44) are feasible with $\lambda_{2}=-1.382, \lambda_{5}=-4$, that means for any time delay function $\tau(t)$ satisfying $0 \leq \tau(t) \leq 0.2$ and $\dot{\tau}(t) \leq 0.1$, the system (4.1) achieves phase synchronization. In the following, we give the simulation results for the case of the time delay function $\tau(t)=$ $0.05 \sin (t)+0.051$, and obviously $\tau(t)$ satisfies $0 \leq \tau(t) \leq 0.2$ and $\dot{\tau}(t) \leq 0.1$. The difference between state variables $x_{i 1}$ and $x_{i m}(i=1,2, m=2, \ldots, 5)$ and the phase difference between $\sigma_{1}$ and $\sigma_{m}(m=2, \ldots, 5)$ are shown in Figure 2. And we can get that the state error variables are convergent to zero as $t \rightarrow+\infty$, and the phase error variables are convergent to zero and $2 \pi$ 


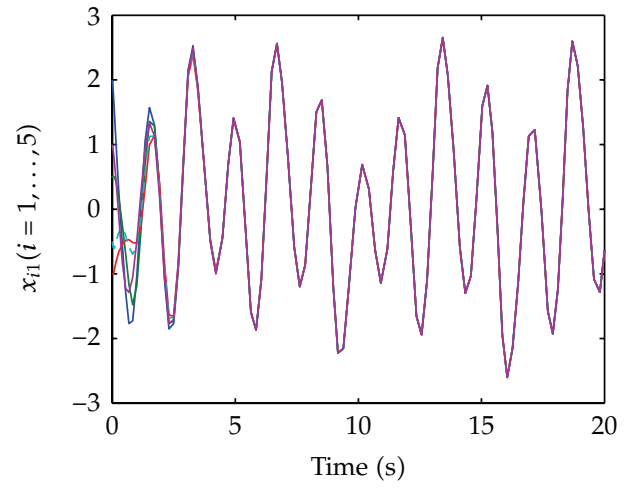

(a)

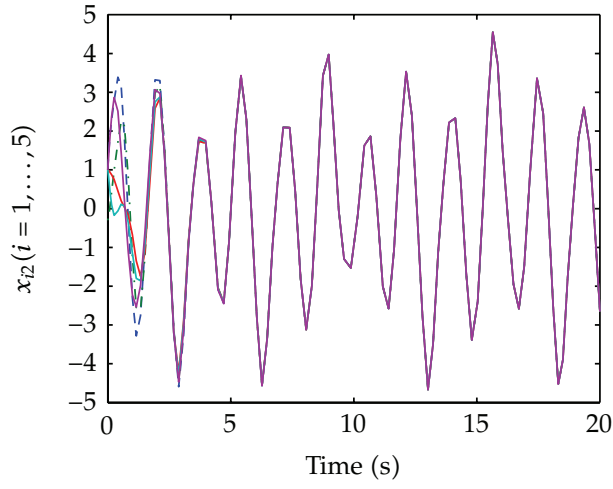

(b)

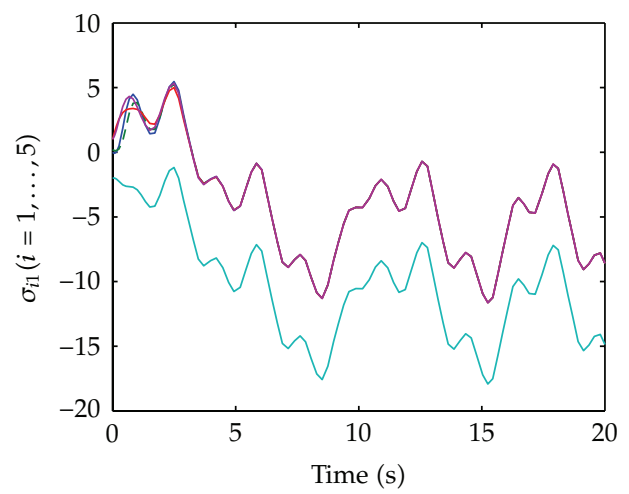

(c)

Figure 3: The synchronization variations in (4.1) of $x_{i 1}, x_{i 2}$, and $\sigma_{i}, i=1, \ldots, 5$.

as defined by Corollary 3.5, which illustrated that the complex network (4.1) achieves phase synchronization. The changes of the synchronous states $x_{i 1}(t), x_{i 2}(t), \sigma_{i}(t) \quad(i=1, \ldots, N)$ are shown in Figure 3, from which we can also observe that the complex network (4.1) achieves the phase synchronization. All of these illustrate that result coincides with the theorem given above. Hence, the effectiveness of the proposed criterion has been proved.

\section{Conclusion}

In this paper, the effects of interconnections between two independent second-order pendulum-like systems have been investigated. Linear interconnection and a class of input and output interconnections have been involved. Some frequency domain and LMI conditions of dichotomy for interconnected pendulum-like systems have been established. Examples show that input and output interchange presented here can result in great changes in some practical systems. For example, chaotic phenomenon in partial variables may appear by adding interconnections between two independent second-order pendulum-like systems which are dichotomous. Since the solution $\sigma$ is unbounded, there is no chaotic phenomenon in plane phase space. However, chaotic phenomenon appears on the cylindrical surface of cylindrical phase space, here we call it the chaos on cylindrical surface, which was never 
studied by now. It shows the complexity of physical property in concrete systems even they are dichotomous. This also indicates that it is possible for the existence of chaotic attractors in pendulum-like systems.

\section{Acknowledgments}

This work is supported by National Natural Science Foundation of China under Grant no. 60874026 and Natural Science Foundation of Heibei province, China under Grant no. $07 \mathrm{M} 007$.

\section{References}

[1] X. F. Wang, L. Xiang, and G. R. Chen, The Theory and Application of Complex Network, Tsinghua University, Beijing, China, 2006.

[2] G. A. Leonov, D. V. Ponomarenko, and V. B. Smirnova, Frequency-Domain Methods for Nonlinear Analysis. Theory and Applications, vol. 9 of World Scientific Series on Nonlinear Science. Series A: Monographs and Treatises, World Scientific, River Edge, NJ, USA, 1996.

[3] S. Xu and Y. Yang, "Predicting dynamic behavior via anticipating synchronization in coupled pendulum-like systems," Journal of Physics A, vol. 42, no. 33, Article ID 335207, 15 pages, 2009.

[4] S. Xu and Y. Yang, "Global asymptotical stability and generalized synchronization of phase synchronous dynamical networks," Nonlinear Dynamics, vol. 59, no. 3, pp. 485-496, 2010.

[5] C. W. Wu, Synchronization in Coupled Chaotic Circuits and Systems, vol. 41 of World Scientific Series on Nonlinear Science. Series A: Monographs and Treatises, World Scientific, River Edge, NJ, USA, 2002.

[6] C. W. Wu and L. O. Chua, "Application of Kronecker products to the analysis of systems with uniform linear coupling," IEEE Transactions on Circuits and Systems I, vol. 42, no. 10, pp. 775-778, 1995.

[7] S. Boyd, L. ELGhaoui, E. Feron, and V. Balakrishnam, Linear Matrix Inequalities in Systems and Control, SIAM, Philadelphia, Pa, USA, 1994.

[8] Y. Yang, R. Fu, and L. Huang, "Robust analysis and synthesis for a class of uncertain nonlinear systems with multiple equilibria," Systems \& Control Letters, vol. 53, no. 2, pp. 89-105, 2004.

[9] Z. Duan, J.-Z. Wang, and L. Huang, "Special decentralized control problems and effectiveness of parameter-dependent Lyapunov function method," in Proceedings of the American Control Conference (ACC '05), vol. 3, pp. 1697-1702, Portland, Ore, USA, July 2005.

[10] Y. Yang, Z. Duan, and L. Huang, "Nonexistence of periodic solutions in a class of dynamical systems with cylindrical phase space," International Journal of Bifurcation and Chaos in Applied Sciences and Engineering, vol. 15, no. 4, pp. 1423-1431, 2005. 